\title{
Martensitic transformations with a classical isotropic two body potential: Intrinsic hysteresis and superelasticity
}

\author{
M.F. Laguna ${ }^{\text {a }}$, E.A. Jagla \\ Centro Atómico Bariloche and CONICET. Bustillo 9.500, Bariloche, Río Negro, Argentina
}

\begin{abstract}
We constructed an isotropic interaction potential for a set of classical identical particles that displays a discontinuous change of its crystalline ground state when a parameter is varied. We used this model to study some properties associated to martensitic transformations. We concentrate here on the triangular-rombohedral transformation in two dimensions, focusing on the hysteresis of the transition, and present also results for the superelasticity effect.
\end{abstract}

\section{Introduction}

A martensitic transformation is a first order, difussionless, solid to solid phase transformation in which the atomic lattice of the austenite high symmetry phase distort to form a new phase of low symmetry (and usually also low temperature) called martensite. The possibility of find a martensitic transformation in a given system is usually associated to the existence of different kind of particles interacting through rather complex (for instance, angle dependent) potentials [1]. It has not been widely appreciated until recently that even isotropic potentials for identical particles can display multiple stable crystalline structures [2,3], and thus can be appropriate to describe qualitatively martensitic transformations. In many cases this behavior can be obtained by slight variations of the potentials with respect to the 6-12 Lennard Jones prototype. This potential is the most widely known case of the family of spherically symmetric potentials, it was originally introduced to study the interactions between atoms of noble gases, and it always gives rise to compact ground state structures: fcc or bcc in three dimensions or hexagonal (triangular) in two dimensions.

Along this line, we introduce a spherically symmetric two body classical potential that produces different crystalline configurations when some parameter is varied. Upon such a variation the stability of different crystalline configurations can change relatively to each other, and this can produce a martensitic transformation in the system. In Ref. [4] we studied in detail the evolution of the transformation and re-transformation processes, and showed that the 2D triangularrombohedral transformation displays the shape memory effect that is associated to some martensitic transformations. For the case of the triangular-square transformation, the same protocols show again the existence of a martensitic transformation, but the shape memory effect is absent, in accordance with theoretical expectations [5].

In this paper we study the characteristics of the hysteresis at the transition, as well as the so called superelasticity effect. Our work demonstrates that simple interatomic potentials in single-species systems are sufficient to realistically study the phenomena associated with martensitic transformations.

\section{Model}

As a whole, the interaction potential we devised has a repulsive core at short distances and an attractive tail at large distances qualitatively similar to the standard Lennard Jones (LJ) potential. However slight modifications are included that alter the contributions to the energy of second and further neighbors. This allows for the possibility to stabilize structures other than the compact ones. Among the many ways of slightly modify the LJ potential, we have found that the following form has the required properties:

a e-mail: lagunaf@cab.cnea.gov.ar

This is an Open Access article distributed under the terms of the Creative Commons Attribution-Noncommercial License (http://creativecommons.org/licenses/by-nc/3.0/), which permits unrestricted use, distribution, and reproduction in any noncommercial medium, provided the original work is properly cited. 
where:

$$
\mathrm{V}(\mathrm{r})=\mathrm{V}_{0}+\mathrm{V}_{1}+\mathrm{V}_{2}+\mathrm{V}_{3}
$$

$$
\begin{array}{ll}
\mathrm{V}_{0}=\mathrm{A}_{0}\left[1 / \mathrm{r}^{12}-2 / \mathrm{r}^{6}+1\right] & \text { if } \mathrm{r}<1 \\
\mathrm{~V}_{1}=\left[(\mathrm{r}-1)^{2}(\mathrm{r}+1-2 \mathrm{c})^{2} /(\mathrm{c}-1)^{4}\right]-1 & \text { if } \mathrm{r}<\mathrm{c} \\
\mathrm{V}_{2}=-\mathrm{A}_{2}\left[\left(\mathrm{r}-\mathrm{d}_{2}-\mathrm{s}_{2}\right)^{2}\left(\mathrm{r}-\mathrm{d}_{2}+\mathrm{s}_{2}\right)^{2}\right] / \mathrm{s}_{2}{ }^{4} & \text { if } \mathrm{d}_{2}-\mathrm{s}_{2}<\mathrm{r}<\mathrm{d}_{2}+\mathrm{s}_{2} \\
\mathrm{~V}_{3}=\mathrm{A}_{3}\left[\left(\mathrm{r}-\mathrm{d}_{3}-\mathrm{s}_{3}\right)^{2}\left(\mathrm{r}-\mathrm{d}_{3}+\mathrm{s}_{3}\right)^{2}\right] / \mathrm{s}_{3}{ }^{4} & \text { if } \mathrm{d}_{3}-\mathrm{s}_{3}<\mathrm{r}<\mathrm{d}_{3}+\mathrm{s}_{3} \quad \text { (0 otherwise in all cases). }
\end{array}
$$

$\mathrm{V}_{0}$ is the repulsive part of a LJ potential and its weight in the total potential is measured by the parameter $\mathrm{A}_{0}$. The quartic term $V_{1}$ contributes with the attractive part to the total potential. The last two terms $V_{2}$ and $V_{3}$ are fine tuning terms that provide a small minimum of amplitude $A_{2}$ centered at $d_{2}$, and a small maximum of amplitude $A_{3}$ centered at $\mathrm{d}_{3}$. They were adjusted to penalize appropriately the triangular lattice, and/or favoring the martensitically related structure. In consequence, the potential is fully determined by the set of parameters $\mathrm{P}=\left\{\mathrm{A}_{0}, \mathrm{~A}_{2}, \mathrm{~A}_{3}, \mathrm{c}, \mathrm{d}_{2}, \mathrm{~s}_{2}, \mathrm{~d}_{3}, \mathrm{~s}_{3}\right\}$. The set $\mathrm{P}_{1}=\left\{\mathrm{A}_{0}, 0.003,0.01,1.722,0.98,0.04,1.74,0.2\right\}$ drives a triangular-rombohedral $(\mathrm{T}-\mathrm{R})$ transition by changing the parameter $\mathrm{A}_{0}$. We found a critical value $\mathrm{A}_{0}{ }^{\mathrm{c}}=0.067$, above which the minimum energy state corresponds to a triangular $(\mathrm{T})$ structure of lattice parameter close to one. For $\mathrm{A}_{0}<\mathrm{A}_{0}^{\mathrm{c}}$, the minimum energy structure is a rombohedral (R) lattice. See details in Ref. [4]. For the T-R transformation, the fact that the symmetry group of $\mathrm{T}$ contains that of R allows us to define $\mathrm{T}$ as austenite, and $\mathrm{R}$ as the martensite phase. Variants can be thought as originated in distortions of the austenite triangular structure along different (but cristallografically equivalent) directions when the martensite is formed. For quantitative evaluation and visualization purposes, we will need to determine whether some particle in the sample corresponds to the triangular structure (austenite), or to any of the martensite variants. We do this by identifying the distorted side of each triangle and drawing the segment in colour. Then, we use three different colours for the three possible different orientations. Undistorted regions of the sample have no segments added. An example of such construction can be observed in Fig. 1. In order to study numerically the T-R transformation in $2 \mathrm{D}$, we solve the time dependence of the particle coordinates according to the Verlet scheme:

$$
\begin{gathered}
\mathbf{r}(\mathrm{t})=2 \mathbf{r}(\mathrm{t}-1)-\mathbf{r}(\mathrm{t}-2)+\mathrm{dt}^{2}[\mathbf{f}(\mathrm{t}-1)-\mu \mathbf{v}(\mathrm{t}-1)] \\
\mathbf{v}(\mathrm{t})=[\mathbf{r}(\mathrm{t})-\mathbf{r}(\mathrm{t}-1)] / \mathrm{dt}
\end{gathered}
$$

Here, $\mathbf{r}(\mathrm{t})$ is the position of each particle, $\mathbf{v}(\mathrm{t})$ its velocity and $\mathbf{f}(\mathrm{t})$ the total force acting over the particle at time $\mathrm{t}$.

Note that we introduce a local friction term proportional to the velocities. However, we do not incorporate any stochastic forcing term, so our approach can be considered to be a zero temperature Langevin simulation [6]. Our approach efficiently takes out the thermal energy generated during the transition, avoiding the building up of large particle velocities. Protocols depending on the global energy (the Nose-Hoover thermostat, for instance) would not be effective [7]. All the results showed in this work correspond to a system of $\mathrm{N}=40000$ particles with open boundary conditions.

\section{Results}

To reproduce the basics of martensitic transformations using our potential, we prepared a starting single crystal sample of rectangular shape in a triangular structure, and choose a value of $\mathrm{A}_{0}=0.085$ for which the $\mathrm{T}$ structure is the stable one. We relax this configuration through the dynamical algorithm and obtain the relaxed starting configuration in the austenite phase. This configuration is shown in Fig. 1a.

Starting from this relaxed configuration, we decrease the parameter $\mathrm{A}_{0}$ at a constant rate and observe the evolution of the system as the T-R transformation proceeds. Transformation starts by the nucleation of martensite variants that invade the interior of the sample during the transformation process. In this progression to the interior, it is also apparent the existence of two variant wedge sectors that are also ubiquitously observed in real samples (see Fig. 1b). The borders of the wedge correspond to the habit lines of the transformation. In the final configuration the existence of the wedges that were important during the transformation has practically disappeared. At this final stage the existence of twinned martensite is also appreciable. In Fig. $1 \mathrm{c}$ we show the martensitic phase obtained for $\mathrm{A}_{0}=0.051$.

As the next step, we reverse the previous conditions increasing $A_{0}$ and found that the initial arrangement of Fig. 1a is recovered. This result is a consequence of the way in which the T-R transformation proceeds: the displacement of the particles is much lower than the interparticle distance itself during the process, and they have a unique path to come 
back to the initial position. In a related work [4] we showed that this behavior is the main cause of the shape memory effect in our model.

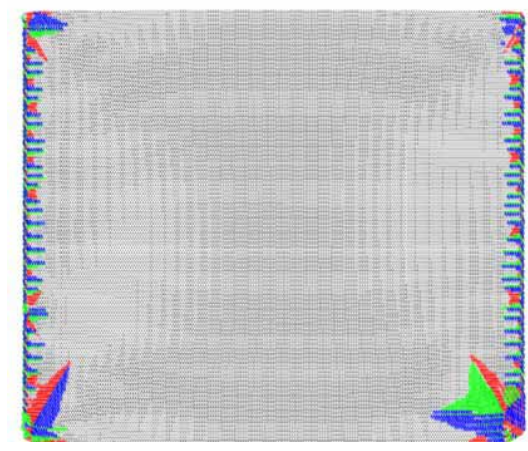

a)

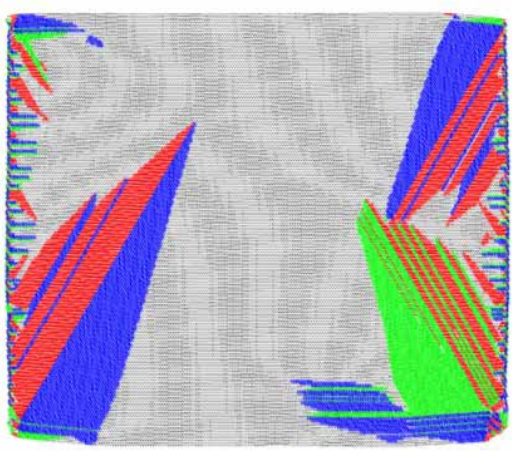

b)

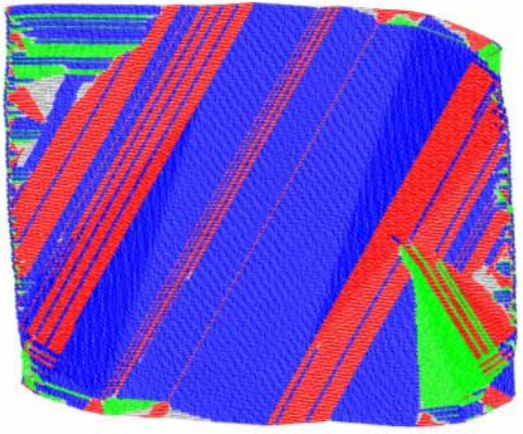

Fig. 1: T-R transformation driven by the change in the parameter $A_{0}$. a) Initial state in austenite phase, relaxed at $A_{0}=0.085$. From this configuration, a constant rate decrease in $\mathrm{A}_{0}$ is applied. b) Intermediate configuration corresponding to $\mathrm{A}_{0}=0.061$. c) Almost completely transformed structure at $\mathrm{A}_{0}=0.051$.

\subsection{Intrinsic Hysteresis}

The behavior of the system during the transformation-retransformation process can be conveniently described by the evolution of the fraction of particles in the martensitic phase, $\mathrm{N}_{\mathrm{m}} / \mathrm{N}$. In Fig. $2 \mathrm{a}$ we show this quantity as a function of $A_{0}$. Black lines of square symbols correspond to the cycle showed in Fig. 1. The shoulder observed in the region $0.073<\mathrm{A}_{0}<0.080$ reflects the fact that a large grain of martensite - the blue one located in the center of the sample in Fig. $1 \mathrm{c}$ - is retained during the retransformation process; only in a later stage it converts to the austenite phase. Red curves with circles correspond to a cycle starting not from the austenite phase but from a martensitic phase formed by small regions of variants (not shown here). In this case, the transformation to the austenite phase proceeds in a single stage, with no retained variants. This result indicates that the shape of the hysteresis loop is strongly dependent on the dynamics of the transformation.

The results shown in Fig. 1a were obtained at a small rate of change of the parameter $\Delta \mathrm{A}_{0}=10^{-9}$ in each time step. To analyze in what extent the width of the hysteresis loop depends on $\Delta \mathrm{A}_{0}$, we followed the same protocol described for Fig. 1 for different values of $\Delta \mathrm{A}_{0}$ to obtain different loop widths, $\Delta \mathrm{H}_{0}$. The results for four different runs are shown in Fig. 2b. To quantify this result we calculated $\Delta \mathrm{H}_{0}$ in each cycle as the difference between the two values of $\mathrm{A}_{0}$ at a fixed value of $\mathrm{N}_{\mathrm{m}} / \mathrm{N}(=0.5)$. The value $\Delta \mathrm{H}_{0}>0$ in the limit $\Delta \mathrm{A}_{0}=0$ measures the intrinsic hysteresis of the transition in the present conditions. For the sample geometry used, this hysteresis is strongly dependent on the nucleation barriers at the surface of the sample. Other geometries may have different intrinsic hysteresis associated to the corresponding facility of nucleation of the martensite phase.

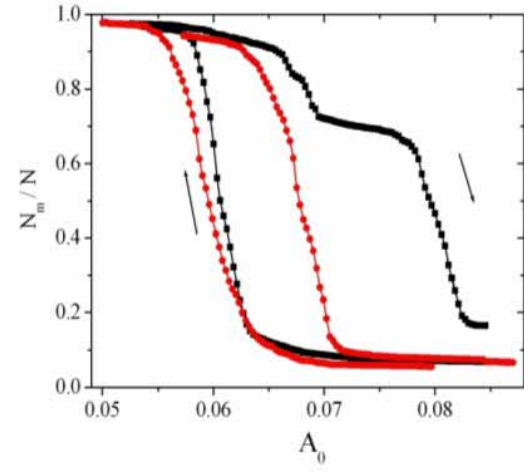

a)

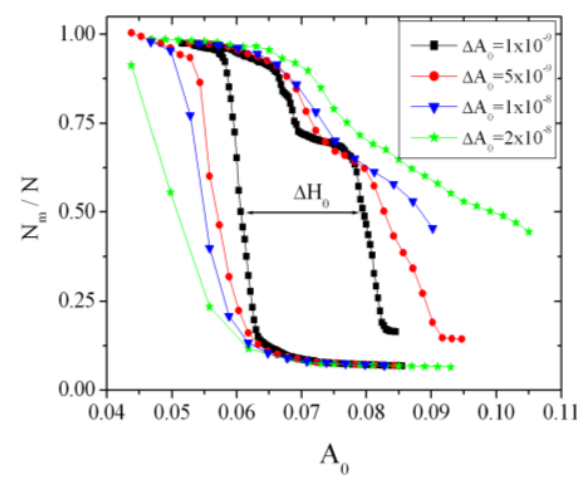

b)

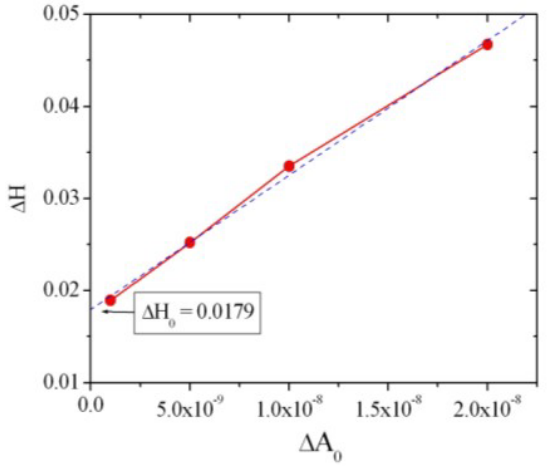

c)

Fig. 2: a) Hysteresis loops displaying the fraction of particles in the martensitic phase as a function of $A_{0}$ for two different initial conditions. b) Hysteresis loops for different velocities of $\mathrm{A}_{0}$ variation. The values of $\Delta \mathrm{A}_{0}$ are indicated in the figure. c) Width of the hysteresis loops as a function of a rate of change of $\mathrm{A}_{\mathrm{o}}$ a finite value for $\Delta \mathrm{A}_{0} \rightarrow 0$ can be inferred. 


\subsection{Stress induced martensitic transformation}

In a related work [4] we verified that this $2 \mathrm{D}$ system displays the shape memory effect. Moreover, here we explore the possibility that superelasticity be also present. We start the process with the sample in the state showed in Fig. 1a and fixed $\mathrm{A}_{0}=0.0675$, a value slightly greater than the critical value $\mathrm{A}_{0}{ }^{\mathrm{c}}=0.067$, to ensure the stability of the austenite phase. We let the system relax and observe no appreciable evolution of the particles. Then, we apply a mechanical stretching on two opposite ends of the sample at the $\mathrm{x}$-direction. This is done by controlling the longitudinal positions of a group of particles in some superficial region of the sample at the two sample ends, and moving this coordinates according to the external forcing conditions. The transverse coordinates of these particles are left to relax according to their normal dynamical evolution, to avoid spurious strain accumulations in the surface sample region.

We observe a first stage in which the system deforms without transforming (the first $5 \times 10^{5}$ time steps), followed by the growth of the martensitic variant that more easily accommodates the deformation, as we show in Fig. 3a. After $2 \times 10^{6}$ steps, almost all the sample is in the martensitic phase and a macroscopic change of shape is observed. The mechanical stretching continues until the time $t=3.4 \times 10^{6}$. During such process the system continuously deforms but the martensitic phase remains stable.

To mimic the experimental protocol, the next stage should be to withdraw the external force and observe that the system recovers the initial state. However, when the system is released, it relax a bit to accommodate the deformations but remains in a state similar to the one of Fig. 3b. Retransformation to the austenite phase only happens if we either shrink the system or slightly increase $\mathrm{A}_{0}$. We presume that this is due to the initial choice of $\mathrm{A}_{0}$, which lays inside the hysteresis curve (see Fig. 2). At the present, simulations are being made with a higher value of $\mathrm{A}_{0}$ outside of the hysteresis region. We expect to observe the retransformation without changing any parameter.

A promising result is that, during the shrinking process, a triangular phase is reobtained with the same lattice parameter as the initial condition.

An interesting behaviour associated to the shrinking process is also observed: if the shrinking continue after the retransformation process to the austenite phase ends, a new T-R transformation occurs. In Fig. 3c we show an intermediate state of this second T-R transformation. At the time showed in the figure, almost all the red martensitic variant disappeared and the other two are growing, as now they are energetically favored.

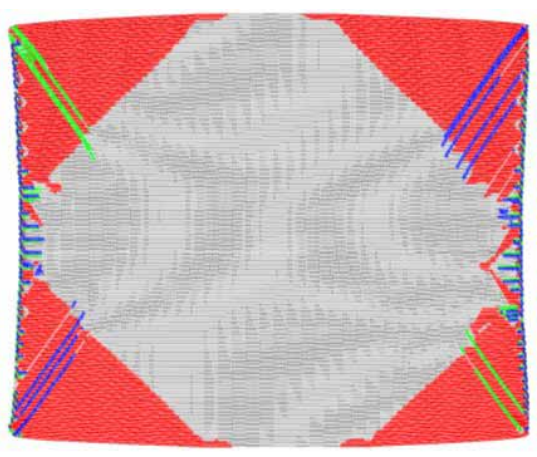

a)

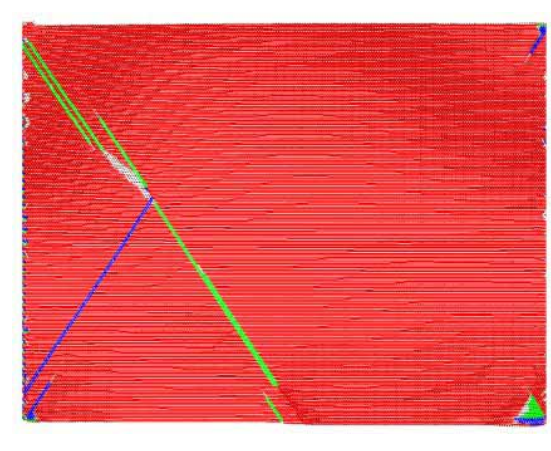

b)

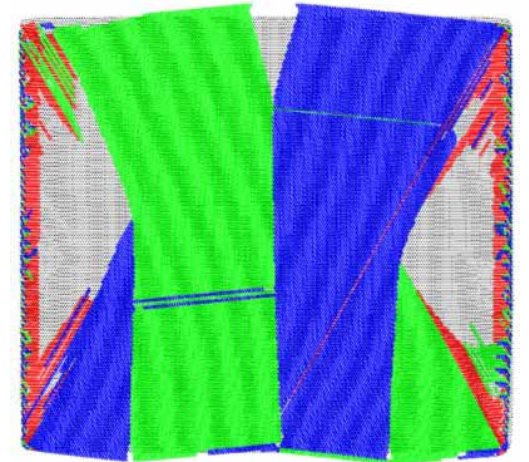

c)

Fig. 3: T-R transformation driven by tension. The initial condition is shown Fig. 1a. a) Intermediate state in in the T-R transformation process after $1.25 \times 10^{6}$ time steps. b) Martensitic phase obtained after $2.47 \times 10^{6}$ time steps. Note that almost all the system is in the same martensitic variant. c) Second T-R transformation, when the system is shrinked.

The full cycling protocol can be conveniently described by two complementary quantities. One measures the overall aspect ratio of the sample and is defined as the ratio Ix/Iy, with $I x=(x(i)-<x>)^{2}$ and $I y=(y(i)-<y>)^{2}$. Its evolution can be seen in Fig 4a and clearly shows the stages previously described. The other is the fraction of the system in each martensitic variant. We shown its evolution in Fig 4b, where the red curve corresponds to the red variant of Fig. 3 and the blue and green curves are the other two martensitic variants.

Note that during the whole period $\mathrm{f}_{\mathrm{x}}>0$ the aspect ratio increases in spite that the transformation ends after $2 \times 10^{6}$ time steps (when the red curve of Fig. $4 \mathrm{~b}$ saturates). In the stage $\mathrm{f}_{\mathrm{x}}=0$ the aspect ratio shows the relaxation of the sample but the fraction of martensitic variants remain almost constant. In the last stage $\left(\mathrm{f}_{\mathrm{x}}<0\right)$ both the aspect ratio and the fraction of red martensitic variant decrease, signaling the retransformation process. 


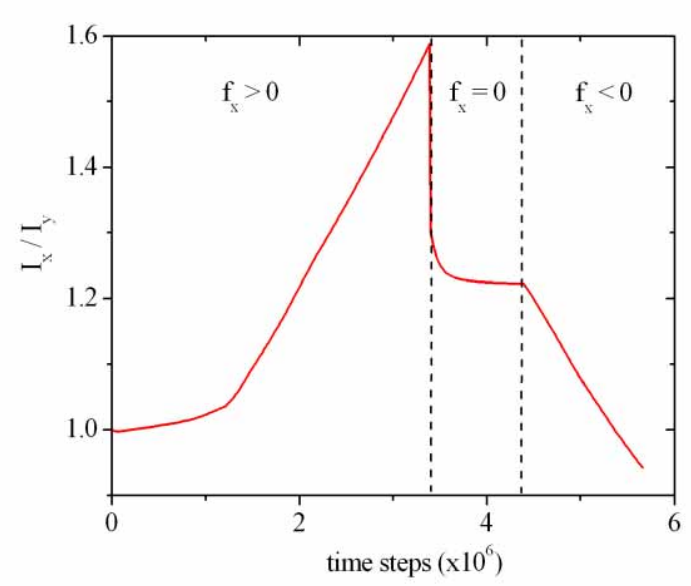

a)

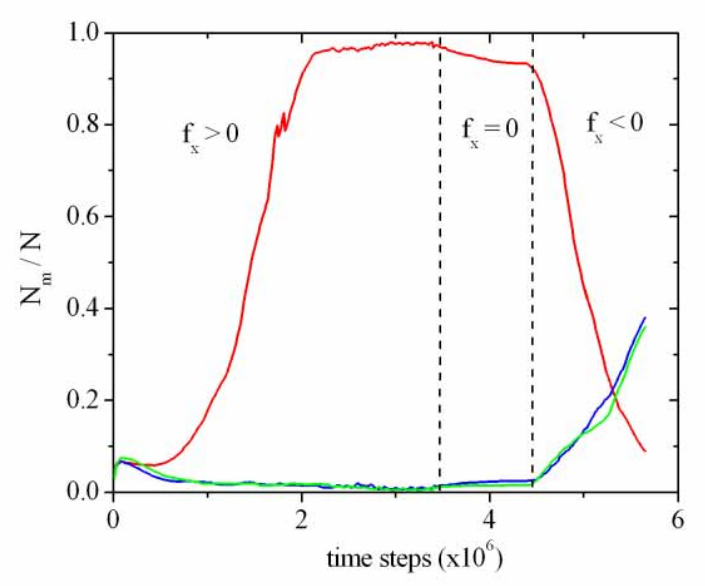

b)

Fig. 4: T-R transformation driven by tension. A positive value of $f_{x}$ indicates the stretching stage, $f_{x}=0$ is the relaxing stage with no force applied. Finally, $\mathrm{f}_{\mathrm{x}}<0$ is the shrinking stage. a) Aspect ratio as a function of time, normalized at the value of the ratio at $\mathrm{t}=0$. $\mathrm{b}$ ) Fraction of particles in the three martensitic variants. Red curve corresponds to the red variant of Fig. 3. Blue and green curves measure the fraction of the other two martensitic variants.

\section{Conclusions}

We use a $2 \mathrm{D}$ isotropic interaction potential between a set of classical identical particles to study some properties associated to martensitic transformations.

We found a set of parameters for the potential for which a hexagonal-rombohedral transformation is present. The hysteresis of the transition was studied for a particular sample geometry, and it was shown that superelasticity could be present in our simple model.

The present and related work [4] demonstrates that simple interatomic potentials in single-species systems can be successfully used to study many phenomena associated with martensitic transformations.

\section{References}

[1] J. Bhattacharya, A. Paul, S. Sengupta and M. Rao; J. J. Phys.: Condens. Matter 20, 365210 (2008).

[2] E. A. Jagla; Phys. Rev. E 58, 1478 (1998).

[3] M. C. Rechtsman, F. H. Stillinger, S. Torquato, Phys. Rev. Lett. 95, 228301 (2005).

[4 M. F. Laguna and E. A. Jagla; Classical isotropic two body potentials generating martensitic transformations. Sent to J.Stat. Mech. (arXiv:0907.3907v1) (2009).

[5] K. Bhattacharya; Microstructure of martensite: why it forms and how it gives rise to the shape-memory effect, Oxford University Press, Oxford, New York (2003).

[6] J.M. Haile; Molecular Dynamics Simulation: Elementary Methods, Wiley, New York, USA (1992).

[7] M. P. Allen, D. J. Tildesley; Computer simulation of liquids, Oxford University Press, Oxford, England (1987). 